\title{
Severity of Liver Involvement in Children with Dengue Infection
}

\author{
Afsana Yasmin ${ }^{1}$, Rozina Akhter ${ }^{2}$, Shahina Pervin Shapla ${ }^{3}$, Laila Yeasmin ${ }^{4}$
}

${ }^{1}$ Registrar, Department of Paediatrics, Dr. Sirajul Islam Medical College and Hospital, Dhaka, Bangladesh; ${ }^{2}$ Associate Professor, Department of Paediatrics, Dr. Sirajul Islam Medical College and Hospital, Dhaka, Bangladesh; ${ }^{3}$ Associate Professor, Department of Paediatrics, Dr. Sirajul Islam Medical College and Hospital, Dhaka, Bangladesh; ${ }^{4}$ Professor \& Chairman, Department of Paediatrics, Dr. Sirajul Islam Medical College and Hospital, Dhaka, Bangladesh

[Received: 12 July 2020; Accepted: 30 August 2020; Published: 1 December 2020]

\begin{abstract}
Background: Liver involvement in dengue infection is one of the clinical presentations. Objective: This study was undertaken to see severity of liver dysfunction in children with dengue infection. Methods: It is a cross sectional observational study conducted in 100 consecutive children with serologically positive dengue infection aged between 2 months to 14 years of age. Liver function tests were done in all patients and were studied the severity of deranged LFT with severity of dengue infection. Results: Out of 100 admitted children with dengue infection, deranged liver function tests found in 57(57.0\%). ALT was above normal in 10 (29.4\% of DF, $19(57.6 \%)$ cases of DHF and 19(57.6\%) cases of DSS respectively. AST was above normal level in 12(35.3\%) cases of DF, 23(69.7\%) cases of DHF and 22(66.7\%) cases of DSS respectively. Low albumin was found in $41.0 \%$ of cases, $15(45.5 \%)$ cases of DHF and 19(57.6\%) cases of DSS. INR was high only 4 cases of DSS. The bilirubin was normal in all cases except one died due to ALF due to DSS. The mean ALT and mean AST statistically higher in DHF and DSS in compare to DF (P-value 0.011 and 0.037 ) respectively. However, there was no significant difference in meal ALT and AST between DHF and DSS respectively. Overall mortality in 2 cases with DHF and DSS. Conclusion: Dengue infection is associated with variable level of liver dysfunction. The severity of liver dysfunction can predict the severity of dengue infection. [Bangladesh Journal of Infectious Diseases, December 2020;7(2):90-94]
\end{abstract}

Keywords: ALT; AST; children; dengue infection; acute liver failure

Correspondence: Dr. Afsana Yasmin, Department of Paediatrics, Dr. Sirajul Islam Medical College and Hospital limited, Dhaka1000, Bangladesh; Email: afsanapgn@gmail.com

Conflict of interest: No conflict of interest

Funding agency: None

Contribution to authors: Yasmin A, Akhter R, Shapla SP, Yeasmin L involved in protocol preparation, data collection and literature search up to manuscript writing.

How to cite this article: Yasmin A, Akhter R, Shapla SP, Yeasmin L. Severity of Liver Involvement in Children with Dengue Infection. Bangladesh J Infect Dis 2020;7(2):90-94

Copyright: (02020. Yasmin et al. Published by Bangladesh Journal of Infectious Diseases. This article is published under the Creative Commons CC BY-NC License (https://creativecommons.org/licenses/by-nc/4.0/). This license permits use, distribution and reproduction in any medium, provided the original work is properly cited, and is not used for commercial purposes. 


\section{Introduction}

Dengue virus infection continues a major health problem in large part of world ${ }^{1}$. The maximum burden of which is borne by the countries of the Asia-Pacific-Region ${ }^{2}$. The presentation of dengue viral illness is diverging from asymptomatic subclinical infection to severe multiorgan involvement and death ${ }^{3}$. Liver involvement in dengue infection is a well-known feature, ranging from mild to moderate elevation of serum transaminases to fulminant liver failure ${ }^{4-9}$.

The last severe outbreak occurred in Bangladesh from May to November 2019. A total 100107 cases and 129 deaths have been officially documented by the government surveillance systems with a clear predominance of cases and fatalities during the summer months (July to November $)^{10}$. DENV 3 was reported is cause of this outbreak of dengue infection in $2019^{11}$. This study was undertaken to see the severity of liver involvement with dengue illnesses in children.

\section{Methodology}

This cross-sectional observational study was conducted in a tertiary care hospital of Dhaka, Bangladesh. A total 100 consecutive children with serologically diagnosed dengue infection aged 2 months to 14 years admitted in pediatric ward and PICU since $25^{\text {th }}$ June to $25^{\text {th }}$ July 2019 was included this study. Data was collected in preformed data collection sheet prospectively. Dengue infection was diagnosed by high-grade fever for few days and serologically confirmed by positive NS1 antigen or IgM ELISA. The laboratory tests like $\mathrm{CBC}$, liver function test, creatinine, electrolytes, calcium and CRP done in all cases. Blood for culture sensitivity was also done in suspected cases of secondary bacterial infection. CXR and ultrasonogram of whole abdomen were done to see, pleural effusion, consolidation and ascites. The study population was divided into three groups Dengue fever (dengue infection without complications, Dengue Hemorrhagic fever (DHF) and dengue shock syndrome (DSS) according to WHO guideline. At the end of study data were analysed by SPSS for
Windows version 22. Descriptive statistics were used for demographic and baseline data and were presented as mean \pm standard deviation (SD), number or percentage. Chi-square test was used for categorical variable. $P$ values $<0.05$ was considered as significant.

\section{Results}

A total 100 consecutive children with dengue virus infection was included in this study. All patients were serologically confirmed dengue, dengue NS1 was positive in $86(86.0 \%)$ children and IgM ELISA positive in $14(14.0 \%)$ children. Of these 100 children, 58 were male and 42 were female. The study included 27 children below 5 years, 51 children between 5 to 10 years and more than 10 years 22 children.

Table 1: Clinical and Laboratory Manifestations of Study Population

\begin{tabular}{|c|c|c|}
\hline Manifestations & Frequency & Percent \\
\hline Fever for $1-5$ days & 100 & 100 \\
\hline Headache, Body aches & 60 & 70 \\
\hline $\begin{array}{l}\text { Nausea, Vomiting, } \\
\text { Abdominal Pain }\end{array}$ & 66 & 66 \\
\hline $\begin{array}{l}\text { Petechiae, Purpura, } \\
\text { Mucosal Bleeding }\end{array}$ & 20 & 20 \\
\hline hepatomegaly & 10 & 10 \\
\hline Ascites & 14 & 14 \\
\hline $\begin{array}{l}\text { Pleural Effusion and /or } \\
\text { Consolidation }\end{array}$ & 36 & 36 \\
\hline $\begin{array}{l}\text { Leukopenia }(<4000 / \text { cum } \\
\mathrm{m})\end{array}$ & 8 & 8 \\
\hline Thrombocytopenia & 80 & 80 \\
\hline$(<1.5 \mathrm{lac})$ & 25 & 25 \\
\hline$<30000$ & 20 & 20 \\
\hline $30000-<50000$ & 27 & 27 \\
\hline $\begin{array}{l}50000-<1 \mathrm{lac} \\
1 \mathrm{lac}-1.5 \mathrm{lac}\end{array}$ & 8 & 8 \\
\hline Hypocalcemia & 19 & 19 \\
\hline $\begin{array}{l}\text { Dyselectrolytemia } \\
\text { - Hyponatrinemia } \\
\text { - Hypokalemia }\end{array}$ & $\begin{array}{c}8 \\
28\end{array}$ & $\begin{array}{c}8 \\
28\end{array}$ \\
\hline Hypoalbuminemia & 41 & 41 \\
\hline
\end{tabular}

Among the study population, 34 were diagnosed as dengue fever, 33 were dengue haemorrhagic fever and 33 were dengue shock syndrome. The 
patients were presented with fever, anorexia, vomiting, abdominal pain, headache, body ache, myalgia and bleeding manifestation. Imaging findings suggestive of ascites in 14 patients and

Table 2: Liver Dysfunction in Dengue Fever

\begin{tabular}{|l|c|c|c|}
\hline Parameters & DF & DHF & DSS \\
& $\mathbf{N}(\mathbf{3 4})$ & $\mathbf{N}(\mathbf{3 3})$ & $\mathbf{N}(\mathbf{3 3})$ \\
\hline ALT & & & \\
$\bullet \quad$ Above Normal (>45U/L) & $10(29.4 \%)$ & $19(57.6 \%)$ & $19(57.6 \%)$ \\
$-\quad>10$ times of normal & $0(0 \%)$ & $5(15.2 \%)$ & $4(12.1 \%)$ \\
\hline AST & & & \\
$\bullet \quad$ Above Normal (> 45 U/L) & $12(35.3 \%)$ & $23(69.7 \%)$ & $22(66.7 \%)$ \\
$-\quad>10$ times of normal & $0(0.0 \%)$ & $6(18.2 \%)$ & $5(15.2 \%)$ \\
\hline Albumin Low & $7(20.5 \%)$ & $15(45.5 \%)$ & $19(57.6 \%)$ \\
\hline INR high & $0(0.0 \%)$ & $0(0.0 \%)$ & $4(12.1 \%)$ \\
\hline
\end{tabular}

pleural effusion and or consolidation was present in 36 children. Leukopenia, Thrombocytopenia, Hypocalcemia, Hypoalbuminemia and Dyselectrolytemia were found in 8, 80, 19, 41 and 36 children respectively. Serum creatinine was high only in $3.0 \%$ of patient. Lowest platelet below 30000 was found in 25 children. Two cases had died. One was a 3.5 years old boy a case of DSS with acute liver failure. His bilirubin 5.9 $\mathrm{mg} / \mathrm{dl}$, ALT was 3073 and AST 3670, INR 2.7, albumin 2.5, creatinine $1.3 \mathrm{mg} / \mathrm{dl}$ and lowest platelet 28000 per cumm. Other one was a 3 years old boy case of DHF with acute liver failure with hepatic encephalopathy stage IV. His bilirubin 0.7 mg/dl, ALT 1213 and AST 3820, INR 1.8, alb 3, platelet 21000, CRP high and blood c/s staphylococcus positive. Blood culture was positive in another patient who was improved.

Table 3: Difference of mean ALT and AST between DF and DHF and DF and DSS

\begin{tabular}{|l|l|l|l|}
\hline \multicolumn{2}{|l|}{ Parameters } & Mean $( \pm$ SD $)$ & P value \\
\hline \multirow{2}{*}{ ALT } & DF & $59.6( \pm 73.4)$ & 0.011 \\
\cline { 2 - 3 } & DHF & $242.4( \pm 383.6)$ & \\
\hline \multirow{2}{*}{ AST } & DF & $68.5( \pm 80.7)$ & 0.037 \\
\cline { 2 - 3 } & DSS & $383.8( \pm 815.4)$ & \\
\hline \multirow{2}{*}{ ALT } & DHF & $242.4( \pm 383.6)$ & 0.495 \\
\cline { 2 - 3 } & DSS & $348.6( \pm 801.5)$ & \\
\hline \multirow{2}{*}{ AST } & DHF & $383.8( \pm 815.4)$ & \multirow{2}{*}{0.946} \\
\cline { 2 - 3 } & DSS & $398.4( \pm 901.9)$ & \\
\hline
\end{tabular}

Bangladesh J Infect Dis
We have done liver function test in all patients. Mean ALT (216 U/L) is lower than mean AST $(281 \mathrm{U} / \mathrm{L})$ in dengue fever. We found deranged LFT more common in DHF and DSS than classical DF. The ALT more than 10 times of normal in (15.2\%) in DHF and $12.1 \%$ in DSS respectively. The AST more than 10 times of normal in $18.2 \%$ in DHF and $15.2 \%$ in DSS respectively. The hypoalbuminemia was also common in DHF and DSS. The bilirubin was high only in 3 patients (Table 2).

Table 3 showed that there was statistically significant difference in mean ALT and mean AST in between DF and DHF and DF and DSS. However, there was no significant difference regarding mean ALT and AST between DHF and DSS.

\section{Discussion}

At present, Dengue is the most rapidly spreading arboviral disease in the world. Approximately, 390 million dengue infections occur annually ${ }^{12}$. In Indian subcontinent, the pattern of dengue infection changes for last few decades from sporadic to endemic form 13 . The dengue circumstances in Bangladesh were getting worse in the year $2019^{14}$. Varying degree of acute liver injury is common in dengue viral infections, but does not associate with the degree of viraemia or the onset or extent of fluid leakage. Dengue associated liver injury appears to peak around day 
6 and 7 or onwards ${ }^{15}$. The degree of liver dysfunction in children with dengue infection varies from mild injury with elevation of transaminases to severe injury with jaundice and acute liver failure. The manifestations of liver involvement such as nausea, vomiting, pain in the right hypochondrium, hepatomegaly, varying degrees of jaundice and an increase in liver markers principally AST and ALT. The previous studies showed liver dysfunction is more common in DSS and DHF than classical dengue fever ${ }^{16-21}$. Previous studies also showed, acute liver failure might occur in severe dengue fever and without intervention fatality rate high ${ }^{22-24}$.

The present study showed $66 \%$ of dengue children are DHF and DSS. ALT elevated in 57.6\% and $57.6 \%$ in DHF and DSS respectively. AST elevated $69.7 \%$ and $66.7 \%$ in DHF and DSS respectively. ALT and AST also elevated in DF in $29.4 \%$ and $35.3 \%$ respectively. ALT and AST are very high more than 10 times of normal in DHF and DSS but none increases such level in DF. Mean ALT and AST significantly higher in DHF and DSS in compare to DF.

DeSouza $^{7}$ and colleagues observed alterations of AST and ALT levels in $63 \%$ and $45 \%$ of patients' respectively. They noted that the average levels of AST and ALT were significantly higher in DHF and DSS patients than in DF patients, an observation supported by other studies. Findings are almost similar with the present study. Mean AST level is higher than mean ALT which is opposite in viral hepatitis.

Bilirubin is high only in 3 patients in this study. Fernando $\mathrm{S}$ et al also found in their study cohort, only 2 pts had elevated bilirubin as cholestatis or biliary stasis, did not significantly occur in dengue associated liver disease ${ }^{15}$. Hypoalbuminemia is common in DHF and DSS ${ }^{15}$. The present study also found 15 and 19 children had hypoalbuminemia in DHF and DSS respectively. Low albumin is usually occurring due to severe liver dysfunction from hypovolumic ischemia or direct viral attack or cytokine mediated injury ${ }^{25}$. INR is high in 4 children with dengue infection.

Laoprasopwattana et $\mathrm{al}^{22}$ showed in Thai children $1.1 \%$ children with dengue virus infection developed acute liver failure and among the ALF cases $68.3 \%$ had died. In the present study of small cohort, we also found $2 \%$ children developed liver failure. One DSS and another DHF with hepatic encephalopathy stage IV and both have died. One of them was superadded with secondary bacterial infection. Profound shock with multiorgan failure, especially involving respiratory failure and/or active bleeding, may be the major cause of death in patients with $\mathrm{ALF}^{25-27}$. Along the liver dysfunction, other complications are found like leucopenia, thrombocytopenia, hypocalcemia, dyselectrolytemia, lung consolidation, pleural effusion, and ascites. One case also developed cardiac complication like myocarditis and arrhythmia. Early recognition of myocardial involvement in dengue illness, prompt restoration of hemodynamic instability while avoiding fluid overload, and sparing unnecessary invasive management are important in treating dengueaffected patients with severe myocarditis ${ }^{26}$. The limitation of the study are small sample size.

\section{Conclusion}

Liver dysfunction is common in children with dengue virus infection. It may be mild hypertransaminesemia to fulminant liver failure. AST level is higher than ALT in dengue infection. Hypoalbuminemia also common in dengue infection may aggravate shock. Jaundice is unusual until development of cholestasis or severe liver failure. Acute liver failure along with encephalopathy or bleeding may causes death. Therefore, early diagnosis and prompt intervention can prevent complications and death.

\section{References}

1. World Health Organization Dengue: guidelines for diagnosis, treatment, prevention and control. World Health Organization, 2009

2. National Guideline for Management of Dengue Syndrome. Disease Control Division, Directorate General of Health Services. Ministry of Health and family welfare, Dhaka, Bangladesh. Third edition 2013.

3. Wilder-Smith A, Schwartz E. Dengue in travelers. N Engl J Med. 2005;353:924-32

4. Karoli R, Fatima J, Siddiqi Z, Kazmi KI, Sultania AR. Clinical profile of dengue infection at a teaching hospital in North India. J Infect Dev Ctries. 2012;6:551-4

5. Saha AK, Maitra S, Hazra SC. Spectrum of hepatic dysfunction in 2012 dengue epidemic in Kolkata, West Bengal. Indian J Gastroenterol. 2013;32: 400-3

6. Trung DT, le Thao TT, Hien TT, Hung NT, Vinh NN, Hien PT, et al. Liver involvement associated with dengue infection in adults in Vietnam. Am J Trop Med Hyg 2010; $83: 774-80$ 
7. DeSouza LJ, Alves JG, Nogueira RM, Gicovate Neto C, Bastos DA, Siqueira EW, et al. Aminotransferase changes and acute hepatitis in patients with dengue fever: analysis of 1,585 cases. Braz J Infect Dis. 2004;8:156-63

8. Kuo CH, Tai DI, Chang-Chien CS, Lan CK, Chiou SS, Liaw YF. Liver biochemical tests and dengue fever. Am J Trop Med Hyg. 1992;47:265-70

9. Nath P, Agrawal DK, Mehrotra RM. Ultrastructural changes in skeletal muscles in dengue virus-infected mice. J Pathol 1982;136: 301-5

10. Mamun MA, Misti JM, Griffiths MD, Gozal D. The dengue epidemic in Bangladesh: risk factors and actionable items. The Lancet. 2019;394(10215):2149-50

11. Shirin T, Muraduzzaman AK, Alam AN, Sultana S, Siddiqua M, Khan MH, et al. Largest dengue outbreak of the decade with high fatality may be due to reemergence of DEN3 serotype in Dhaka, Bangladesh, necessitating immediate public health attention. New microbes and new infections. 2019 May;29

12. Bhatt S, Gething PW, Brady OJ, Messina JP, Farlow AW, Moyes CL, et al. The global distribution and burden of dengue. Nature 2013;496:504-507

13. Gupta N, Srivastava S, Jain A, Chaturvedi UC. Dengue in India. Indian J Med Res 2012;136: 373-390

14. Akram A. Alarming Turn of Dengue Fever in Dhaka City in 2019. Bangladesh J Infect Dis 2019;6(1):1-2

15. Fernando S, Wijewickrama A, Gomes L, Punchihewa CT, Madusanka SD, Dissanayake H, et al. Patterns and causes of liver involvement in acute dengue infection. BMC infectious diseases. 2016;16(1):319

16. Tirumani HB, Bejugam VK, Naseem A, Hussain A et al. Study of hepatic involvement in children with dengue infection. Int J Contemp Pediatr. 2017;4(6):2012-2014

17. Mohan B, Patwari AK, Anand VK. Hepatic dysfunction in childhood dengue infection. J Trop Pediatr. 2000;46:40-43
18. Pancharoen C, Rungsarannont A, Thisyakorn U. Hepatic dysfunction in dengue patients with various severity. J Med Assoc Thai. 2002;85(Suppl 1):S298-301

19. Wahid SF, Sanusi S, Zawawi MM, Ali RA. A comparison of the pattern of liver involvement in dengue haemorrhagic fever with classic dengue fever. Southeast Asian J Trop Med Public Health. 2000;31:259-263

20. Itha S, Kashyap R, Krishnani N, Saraswat VA, Choudhuri G, Aggarwal R. Profile of liver involvement in dengue virus infection. Natl Med J India 2005;18:127-130

21. Chongsrisawat V, Hutagalung Y, Poovorawan Y. Liver function test results and outcomes in children with acute liver failure due to dengue infection. Southeast Asian J Trop Med Public Health. 2009;40:47-53

22. Laoprasopwattana K, Jundee P, Pruekprasert P, Geater A. Outcome of severe dengue viral infection-caused acute liver failure in Thai children. Journal of tropical pediatrics. 2016;62(3):200-5

23. Arora S, Nathaniel SD, Paul JC, Hansdak SG. Acute liver failure in dengue hemorrhagic fever. Case Reports 2015; 2015:bcr2015209443.

24. Dalugama C, Gawarammana IB. Dengue hemorrhagic fever complicated with acute liver failure: a case report. Journal of medical case reports. 2017;11 (1):341

25. Trung DT, le Thao TT, Hien TT, Hung NT, Vinh NN, Hien PT, et al. Liver involvement associated with dengue infection in adults in Vietnam. AmJTrop Med Hyg. 2010;83(4):774-80

26. Lee K, Lee WH, Liu JW, Yang KD. Acute myocarditis in dengue hemorrhagic fever: a case report and review of cardiac complications in dengue-affected patients. International Journal of Infectious Diseases. 2010;14(10):e919-22

27. Nguyen TL, Nguyen TH, Tieu NT. The impact of dengue haemorrhagic fever on liver function. Res Virol 1997;148: 273-7 